\title{
On the primefulness of local cohomology modules
}

\section{Payman Mahmood Hamaali*, Adil Kadir Jabbar}

Department of Mathematics, College of Science, University of Sulaimani (Payman.hamaal@univsul.edu.iq)* (adil.jabbar@univsul.edu.iq)

\section{Article Information}

Received: 10/02/2021

Accepted: 18/03/2021

\section{Keywords:}

Local cohomology

modules, Minimax

modules and Primeful

modules

\begin{abstract}
Let $R$ be a commutative Noetherian ring with identity $1 \neq 0$. For a non-zero $R$-module $M$. We prove that a multiplication primeful $R$-module $M$ and $H_{R}^{k}(M)$ are I-cofinite and primeful, for each $k>0$ where $I$ is an ideal of $R$ with $I \subseteq A n n(M)$. As a consequence, we deduce that, if $M$ and $N$ are multiplication primeful R- modules, then $\operatorname{Ext}_{R}^{k}(M, N)$ is primeful. Another result is, for a projective $R-$ module $M$ over an integral domain, $M$ admits projective resolution $P^{+}$such that each $P_{i}$ is primeful (faithfully flat).
\end{abstract}

\section{Introduction:}

In this paper, $R$ is a commutative Noetherian ring with identity $1 \neq 0$ until otherwise stated and $M$ is an non-zero $R$-module. A submodule $N$ of an $R$-module $M$ is called prime, if $r m \in N$, for each $r \in R$ and $m \in M$ then $m \in N$ or $r \in\left(N:_{R} M\right)$, in this case $\left(N:_{R} M\right)$ is a prime ideal of $R$ and $N$ is called $p$-prime. Consider $\rho: \operatorname{Spec}(M) \rightarrow \operatorname{Spec}(R / \operatorname{Ann}(M))$ such that $\rho(P)=(P: M) / \operatorname{Ann}(M)$ for all $P \in \operatorname{Spec}(M)$ is called the natural map of $\operatorname{Spec}(M)$ [5]. A nonzero $R$-module $M$ is called primeful if $\rho$ is surjective. Chin Pi Lu [5, Theorem 2.2] showed that every finitely generated $R$ - module is primeful but the converse is not true in general, for example every infinite dimensional vector space is primful.

A Primeful $R$-modules are generalization of finitely generated R-modules. Many results for finitely generated modules are generalized to primefuls, the most important one is the Naykayama,s Lemma and the equality $\operatorname{Supp}(M)=V(\operatorname{Ann}(M))$ for $M$ [5].

It is well-known that, if $F=\left\{F^{k}, \alpha^{k}\right\}$ is a cochain complex, then $H_{R}^{k}(M)=$ $\operatorname{Ker} \alpha^{k} / \operatorname{Im} \alpha^{k-1}$ is $k$-th cohomology module of $F$ [4]. The k-th local cohomology module of $M$ with respect to an ideal $I \subseteq A n n(M)$ is $\lim _{k} E x t_{R}^{k}\left(R / I^{k}, M\right)$ [2]. An $R$-module $M$ is called $I$-cofinite if $\operatorname{Supp}(M) \subseteq V(I)$ and $\operatorname{Ext}_{R}^{k}(R / I, M)$ is finitely generated for all $k$ [2]. 
On the other hand, Sean Sather-Wagstaff [6] proved that if $R$ is a commutative ring and $M$ is an R-module, then $M$ admits a free (hence projective) resolution $P^{+}$over R. Also if $M$ is finitely generated then each $P_{i}$ of $P^{+}$is finitely generated over $\mathrm{R}$.

The main purpose of this article, is changing the direction of study by using cohomolgy facts. We prove that, if $M$ is a projective module over an integral domain, then $M$ admits a free (hence projective) resolution $P^{+}$over R such that each $P_{i}$ is primeful (faithfully flat ) over R. In [5] it is shown that a submodule of primeful module need not be primeful. One of the results in this paper, is if we have PID then the following are equivalent for a projective module $P$ :

1- $P$ is projective,

2- $P$ is primeful,

3- There exist a primeful module such that every submodule is primeful.

In section two we give a condition that help $\operatorname{Ext}_{R}^{i}\left(\frac{R}{I}, \Gamma_{I}(M)\right)$ and $\operatorname{Tor}_{i}^{R}\left(R / I, \Gamma_{I}(M)\right)$ for a primeful R-module to be primeful for all i.

\section{The Results}

In this section, we prove that if $M$ is an R-module over PID, then $M$ admits free(hence projective )resolution $P^{+}$over $\mathrm{R}$ such that each $P_{i}$ is primeful over R. Also find a primeful module that every submodule of it is primeful.

Lemma 2.1. Let $M$ be a projective module over an integral domain, then $M$ admits a free (hence projective) resolution $P^{+}$over R such that each $P_{i}$ is primeful (faithfully flat ) over R.

Proof. It is well-known that if $R$ is a commutative ring and $M$ is an R-module, then

$M$ admits a free (hence projective) resolution over R.

A projective module over an integral domain is primeful [5, corollary 4.3] .In this case $M$ admits a free(hence projective ) resolution $P^{+}$over $\mathrm{R}$ such that each $P_{i}$ is primeful over $\mathrm{R}$.

An $R$-module $M$ is called multiplication if every submodule $N=I M$ where $I$ is an ideal of $R$.

In [5,Theorem2.2] showed that every finitely generated module $M$ is primeful,consequentely the quotient module $M / N$ for any submodule $N$ of $M$. For a multiplication module we have some other results, we start with Lemma2.2.

Lemma 2.2. Let $M$ be a multiplication R-module and $0 \rightarrow L \rightarrow M \rightarrow N \rightarrow 0$ be a short exact sequence, then $M$ primeful if and only if $L$ and $N$ are primeful.

Proof. Suppose that M is a multiplication primeful module, we consider $L$ as a submodule of $M$ and $N=M / L$, so by[1, proposition 3.8] $M$ is finitely generated and hence $L$ is alo finitely generated which implies that $L$ and $M / L$ are finitely generated hence primeful [5.Theoem 2.2].

Conversely, suppose that $L$ and $\frac{M}{L}$ are primfule then they are finitely generated, so $M$ is also finitely generated which implies that $M$ is primeful.

It is proved that in [1] that a submodule of a primful module need not be primeful. In Theorem 2.3 we give the condition under which a submodule of primeful module is primeful. 
Theorem 2.3. For a projective R-module $P$ over PID $R$ the following are equivalent:

1- $P$ is projective

2- $P$ is primeful

3- There exist a primeful module such that every submodule is primeful.

Proof. Suppose that $P$ is a projective module, then by [1] projective modules over an integral domain is primeful. To prove (3) , it is well known that, for a projective $R$-module there exist a free $R$-module $F$ such that $P$ is a direct sum of $F$ [6]. Now, free modules over PID are primeful and $F=X \oplus P$ which implies that $F, X, P$ and all other submodules of $F$ are primeful.

Recall (Schanuel's Lemma [6]: Let $\mathrm{R}$ be a commutative ring, and let $\mathrm{M}$ be an R-module. Consider two exact sequences

$$
\begin{aligned}
& 0 \rightarrow K \rightarrow P_{t} \rightarrow P_{t-1} \rightarrow \cdots \rightarrow P_{1} \rightarrow P_{0} \rightarrow M \rightarrow 0 \\
& \quad 0 \rightarrow L \rightarrow Q_{t} \rightarrow Q_{t-1} \rightarrow \cdots \rightarrow Q_{1} \rightarrow Q_{0} \rightarrow N \rightarrow 0
\end{aligned}
$$

such that each $\mathrm{Pi}$ and $\mathrm{Qi}$ is projective. Then $\mathrm{K}$ is projective if and only if $\mathrm{L}$ is projective.

Now by using (Schanuel's Lemma) and appling Theorem 2.3 we can prove the following corollary.

Corollary 2.4. Let $R$ be an integral domain. Consider two exact sequence:

$$
\begin{aligned}
& 0 \rightarrow K \rightarrow P_{t} \rightarrow P_{t-1} \rightarrow \cdots \rightarrow P_{1} \rightarrow P_{0} \rightarrow M \rightarrow 0 \\
& \quad 0 \rightarrow L \rightarrow Q_{t} \rightarrow Q_{t-1} \rightarrow \cdots \rightarrow Q_{1} \rightarrow Q_{0} \rightarrow N \rightarrow 0
\end{aligned}
$$

Where each $P_{i}$ and $Q_{i}$ are projective then:

1- $K \oplus Q_{o} \cong L \oplus P_{o}$

2- $K$ is primeful if and only if $\mathrm{L}$ is primeful.

Proof. By (Schanuel's Lemma) we have each $P_{i}$ and $Q_{i}$ are projective and $R$ be an integral domain. Hence 1 and 2 are satisfying.

Proposition 2.5. If $M$ is a multiplication primeful module, then $\operatorname{Ext}_{R}^{i}\left(\frac{R}{I}, \Gamma_{I}(M)\right)$ and $\operatorname{Tor}_{i}^{R}\left(R / I, \Gamma_{I}(M)\right)$ are primeful for all i.

Proof. Directly by Lemma 2.2 .

In [5, Proposition 3.8] it is provide that for a non-zero $R$-module $M$ the following are equivalent:

1- $M$ is finitely generated

2- $\mathrm{M}$ is primeful

3- $\operatorname{Supp}(M)=V(\operatorname{Ann}(M))$

4- $p M: M=p$ for every $p \in V(\operatorname{Ann}(M))$

5- $p M \neq M$ for every $p \in V(\operatorname{Ann}(M))$.

Proposition 2.6. Let $M$ and $N$ be two multiplication primeful modules, then $\operatorname{Ext}_{R}^{i}(M, N)$ is primeful for each $i$.

Proof. Since we have $M$ and $N$ two multiplication primeful modules, hence by [5, proposition 3.8] they are finitely generated .On the other hand,[ 6, Proposition IV 3.9] shows that for a commutative Noetherian ring, if $M$ and $N$ are finitely generated, then $\operatorname{Ext}_{R}^{i}(M, N)$ is finitely generated for each $i$. Thus by [5,Theorem 2.2] $\operatorname{Ext} t_{R}^{i}(M, N)$ is primeful for each $i$. 
In the following result we provide a condition under which a primefule R-module $M$ and the local cohomology $H_{R}^{i}(M)$ are an $I$-cofinite for each $i$.

Proposition 2.7. Suppose that $M$ is a multiplication primeful R-module, then $M$ and the local cohomology $H_{R}^{i}(M)$ are also $I$-cofinites for each $i$.

Proof. By [5, proposition 3.8] , $\operatorname{Supp}(M)=V(\operatorname{Ann}(M))$.

In [5] shown that, if $M$ is a multiplication module then primeful and finitely generated modules are equivalent. Thus $M$ is $I-$ cofinite.

Similar argument is true for $H_{R}^{i}(M)[2]$.

Acknowledgement. The authors are deeply grateful to the referees for their thorough review and highly appreciate the comments and helpful suggestions, which significantly contributed to improving the quality of the publication.

\section{References}

1. Ansari-Toroghy, H., \& Hassanzadeh-Lelekaami, D. (2009). On the Prime Submodules of Primeful Modules. International Journal of Algebra, 3(3), 119-123.

2. Divaani-Aazar, K., \& Mafi, A. (2005). Associated primes of local cohomology modules. Proceedings of the American Mathematical Society, 133(3), 655-660.

3. Mafi, A. (2011). On the local cohomology of minimax modules. Bulletin of the Korean Mathematical Society, 48(6), 1125-1128.

4. Paul E., (2011), Rings and Their Modules. Walter de Gruyter GmbH \& Co. KG, Berlin, New York.

5. Lu, C. P. (2007). A module whose prime spectrum has the surjective natural map. Houston Journal of Mathematics, 33(1), 125.

6. Sather-Wagstaff, S., (2009), Homology algebra notes. Department of Mathematics, 300 Minard Hall, North Dakota State University, Fargo, North Dakota 58105-5075, USA. 


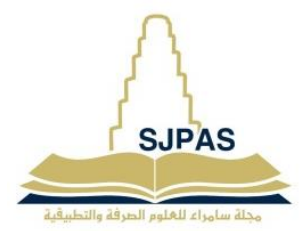

حول بدائية المقاسات الكوهومولوجية المحلية

به يمان محمود حمه علي، عادل قادر جبار قسم الرياضيات، كلية العلوم، جامعة السليمانية عالية

الخلاصة:

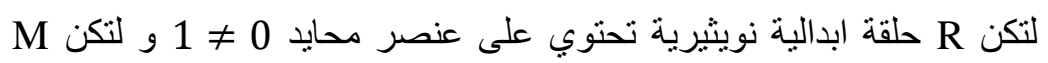

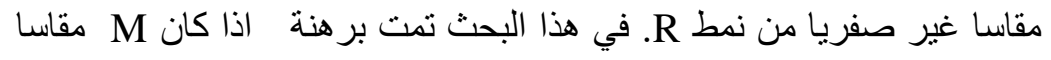
جدائيا وبدائيا فان M و $M$ و

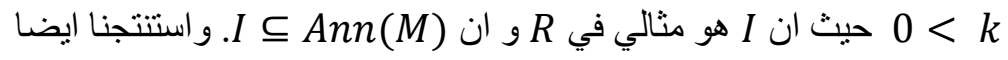

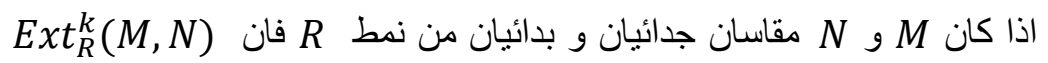

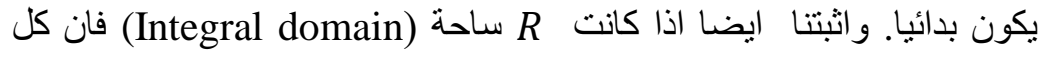
مقاس اسقاطي M من نمط R يقبل بحل اسقاطي +. معلومات البحث:

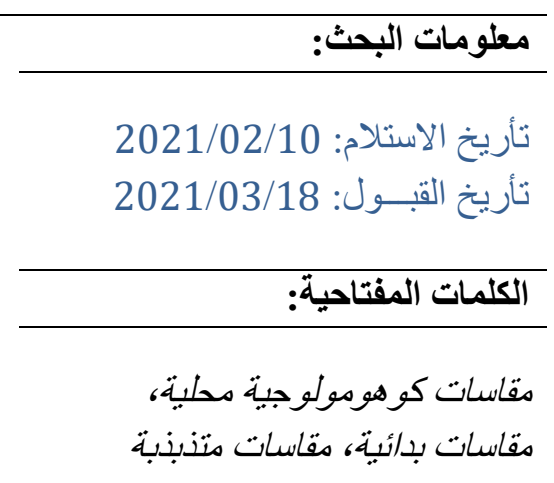

$$
\text { بدائيا (مسطحة بو لاء). }
$$

